\title{
Land-Cover Evolution Class Analysis in Image Time Series of Landsat and Sentinel-2 Based on Latent Dirichlet Allocation
}

\author{
Daniela Espinoza-Molina, Reza Bahmanyar, and Mihai Datcu \\ Remote Sensing Technology Institute (IMF) \\ German Aerospace Center (DLR) \\ Wessling, Germany \\ \{daniela.espinozamolina;reza.bahmanyar;mihai.datcu\}@dlr.de
}

\author{
Ricardo Díaz-Delgado and Javier Bustamante \\ Remote Sensing and GIS Laboratory (LAST-EBD) \\ Estacion Biologica de Doñana \\ Sevilla, Spain \\ \{rdiaz;jbustamante\}@ebd.csic.es
}

\begin{abstract}
Satellite Image Time Series (SITS) are widely used in monitoring the Earth's changes for various applications such as land-cover evolution analysis. In this paper, we propose an approach based on Latent Dirichlet Allocation (LDA) which considers spatial and spectral information to measure the landcover changes in multispectral SITS. For our experiments, we focus on the vegetation dynamics of the Donana National Park (in southwestern Spain) using a Landsat and a Sentinel-2 SITS dataset. The proposed approach represents each image by Normalized Difference Vegetation Index (NDVI) and tiles it into smaller patches. The patches are then modeled as Bag-of-Words (BoW) and LDA is applied to them in order to discover the latent structure of the image. The divergence between the latent structures of any two consecutive images is then considered as the measure of change. Results show that the changes measured by the proposed approach can represent the vegetation dynamics of the region of interest. Moreover, comparing the results obtained from the two datasets demonstrates that using highlevel information allows the proposed approach to measure the changes independent of the sensor. This will support long-term monitoring through combining various available data.
\end{abstract}

\section{INTRODUCTION}

The availability of Satellite Image Time Series (SITS) has broadened their applications to a wide variety of research domains in recent years. They reveal the Earth's changes over time which allows monitoring, analyzing, and characterizing the dynamics of various phenomena over the years. A number of previous research studies in land-cover and landuse analysis have demonstrated the necessity of long-term SITS for characterizing natural variability trends in seasonal to decennial scales [1]-[3]. Díaz-Delgado et al. [1] used a longterm time series of Landsat-5 and Landsat- 7 images during 1974 to 2014 for assessing the hydroperiod trends of the marshes (i.e., a wetland) in Doñana National Park. Throughout their research, various maps have been created and validated based on several in-situ campaigns. Tan et al. [2] applied a classification-based approach to a long-term SITS dataset for monitoring land-cover and land-use dynamics over Zigui County, China. Costachioiu et al. [3] showed the benefits of long-term SITS (over 25 years) in urban development

978-1-5386-3327-4/17/\$31.00 (C)2017 IEEE monitoring. They classified intra-annual image stacks in a supervised manner and compared the results to population dynamics.

Recently, the European Commission has founded an Earth Observation (EO) programme, namely Copernicus, in order to ensure the continuity between operational missions and to provide reliable and up-to-date information for supporting long-term global environment and security monitoring. The operational needs of Copernicus are met through the launching of the Sentinel missions, constellations of satellite pairs. For example, the Sentinel-1 mission, providing Synthetic Aperture Radar (SAR) data acquisitions with different spatial resolutions (from $5 \mathrm{~m}$ to $30 \mathrm{~m}$ ), attempts to provide continuity to the ENVISAT mission that lost contact in 2012 [4]. In the case of optical imagery, the Sentinel-2 mission, offering multispectral images with 13 spectral bands in different spatial resolutions (from $10 \mathrm{~m}$ to $60 \mathrm{~m}$ ), aims at supporting the Landsat mission [5] which is providing images with various spatial resolutions (from $30 \mathrm{~m}$ to $120 \mathrm{~m}$ ) since 1972 .

In order to benefit from the continuity and complementarity of the data provided by such missions, developing approaches which are less sensitive to the different properties of the sensors being used (e.g., resolution, spectra), is in high demand. Ye et al. [6] proposed an approach for SAR SITS classification based on Latent Dirichlet Allocation (LDA) generative model [7] and characterized the images by their latent semantic structures. They established a multilayer model between the low-level image features and the high-level scene to learn the latencies which are more robust against acquisition-related dissimilarities between different images of a scene.

In this paper, we propose an approach for measuring the land-cover changes in multispectral SITS based on LDA. It considers spatial and spectral information to discover the images' latent structures which are then used to compute the changes in the land-covers over time. Our experiments are focused on the vegetation dynamics of the Doñana National Park due to the essential role of its marshlands in western Europe's ecosystem [1]. In order to evaluate the generalizability of our proposed approach for SITS datasets from 
different sensors in support of the continuity missions, we use a Landsat and a Sentinel-2 SITS dataset. Results show that the changes measured by the proposed approach are able to represent the vegetation dynamics of the study area. Additionally, comparing the results obtained from the two datasets demonstrates that using high-level information allows measuring the changes regardless of the sensor. This will help long-term monitoring through the combination of various available data (e.g., continuity missions).

\section{DATASET DESCRIPTION}

Our study zone is the protected area of Doñana National Park in southwestern Spain. The Doñana marshes are considered as the largest sanctuary for migratory birds in western Europe. Therefore, monitoring its land-cover changes is in high demand by decision-makers for an effective management of the park [1], [8]. The Landsat and Sentinel-2 SITS datasets used in our experiments are based on the WGS84 coordinate reference system. The images are located between the northwestern coordinates $(-6.905383 \mathrm{~W}, 37.221228 \mathrm{~N})$ and the southeastern coordinates $(5.957231 \mathrm{~W}, 36.76 \mathrm{~N})$. The Landsat dataset is composed of 18 multispectral images (nine Landsat5 and nine Landsat- 7 images), of $3030 \times 1474$ pixels (the bands with $30 \mathrm{~m}$ spatial resolution), acquired between September 2009 and August 2010. The Sentinel-2 dataset contains 14 multispectral images of the size $9362 \times 4554$ pixels (the bands with $10 \mathrm{~m}$ spatial resolution), acquired from December 2015 to December 2016. Both datasets cover periods of one year allowing to study seasonal land-cover changes.

\section{Methodology}

Multispectral images allow representing the most relevant information to every application through spectral indices, combinations of spectral bands $\left(b_{s}\right)$ validated based on the reflectance properties of the features of interest. In this paper, we represent the multispectral images by Normalized Difference Vegetation Index (NDVI), a green vegetation indicator, in order to study land-cover dynamics. While for the Landsat images $N D V I=\frac{b 4-b 3}{b 4+b 3}$, for the Sentinel-2 images $N D V I=\frac{b 8-b 4}{b 8+b 4}$.

LDA is a probabilistic generative model which discovers the latent structures of data collections as sets of topics [7]. In our approach, LDA is applied separately to the NDVI representation of each image to discover $K$ topics, where $K=7$ based on a scientific guess. The topics are then used for computing the changes between the images.

\section{A. Applying LDA to the Images}

The NDVI representations of the images are split into smaller patches so that the corresponding patches of the Landsat and the Sentinel-2 images cover the same area on the ground. In our experiments, the patch sizes of $9 \times 9$ and $27 \times 27$ pixels were selected for the Landsat and Sentinel-2 images, respectively, due to their different spatial resolutions. These patch sizes are compromises between small patches keeping the semantic analysis simple and bigger patches capturing the spatial context of objects. Then, we compute the local primitive features of each image patch by vectorizing a neighborhood of $3 \times 3$ pixels around every pixel which results in a set of nine-dimensional feature vectors. After that, for each dataset, $1 \%$ of all the computed feature vectors are randomly sampled and $k$-means clustering is applied to them in order to generate dictionary of $N_{V}$ visual words, $V=\left\{v_{1}, v_{2}, \ldots, v_{N_{V}}\right\}$. The number of clusters for both datasets was empirically set to 50 .

The patches are then modeled as Bag-of-Words (BoW), where each $d$-th patch of the $i$-th image is represented as sequences of $N_{d}$ visual word-tokens, $\mathbf{w}_{d}^{i}=$ $\left\{w_{1 d}^{i}, w_{2 d}^{i}, \ldots, w_{N_{d} d}^{i}\right\}$, where the visual word-tokens are drawn from $V$. Next, LDA is applied to the BoW models in order to discover the latent structure as a set of topics, $T^{i}=$ $\left\{t_{1}^{i}, t_{2}^{i}, \ldots, t_{K}^{i}\right\}$. The topics are multinomial distributions over the visual words, denoted by $\beta^{i}$. The distribution of the topics within each $d$-th patch is determined by a $K$-dimensional Dirichlet random variable $\theta_{d}^{i}$. In order to generate each $d$ th patch of the image $I_{i}$, LDA chooses $\theta_{d}^{i} \sim \operatorname{Dir}(\alpha)$, where $\alpha$ parametrizes the Dirichlet distribution. Next, for each $n$ th visual word-token, LDA samples a topic for the topictoken $z_{n d}^{i}$ from $\operatorname{Mult}\left(\theta_{d}^{i}\right)$. Then a visual word is picked for $w_{n d}^{i}$ from the multinomial probability distribution over the visual word dictionary conditioned on the selected topic, $p\left(w_{n d}^{i} \mid z_{n d}^{i} ; \beta^{i}\right)$. For estimating the model parameters $\alpha$ and $\beta$, and inferring the posterior distributions $\theta$, LDA uses a variational Expectation Maximization algorithm [7]. Using the model parameters, the probability of each $w_{n d}^{i}$ is:

$$
p\left(w_{n d}^{i} \mid \theta_{d}^{i} ; \alpha, \beta^{i}\right)=p\left(\theta_{d}^{i} ; \alpha\right) \sum_{z_{n d}^{i}} p\left(z_{n d}^{i} \mid \theta_{d}^{i}\right) p\left(w_{n d}^{i} \mid z_{n d}^{i} ; \beta^{i}\right) \text {. }
$$

\section{B. Computing the Changes Using LDA}

For computing the changes between a sequence of two images, the distribution of the generated words by LDA for each $d$-th patch of the earlier image $\left(I_{i}\right)$, which is computed based on Equation 1, is compared to that of its corresponding patch in the later image $\left(I_{i+1}\right)$. To this end, to each image patch its most frequent topic is assigned as:

$$
\mathbf{w}_{d} \rightarrow t_{\max }=\underset{j}{\arg \max }\left\{p\left(t_{j} \mid \theta_{d}\right) p\left(\theta_{d} \mid \alpha\right)\right\}, j \in[1, K] .
$$

Then the divergence of the topic assigned to every $d$-th patch of $I_{i}$ is computed to that of assigned to the corresponding patch of $I_{i+1}$, using Kullback-Leibler divergence [9],

$$
D_{K L}\left(t_{\text {max }}^{i}|| t_{\text {max }}^{i+1}\right)=\sum_{l=1}^{N_{V}} p\left(v_{l} \mid t_{\text {max }}^{i}\right) \ln \frac{p\left(v_{l} \mid t_{\text {max }}^{i}\right)}{p\left(v_{l} \mid t_{\text {max }}^{i+1}\right)} .
$$

$D_{K L}$ is not symmetric as $D_{K L}\left(t_{\max }^{i} \| t_{\max }^{i+1}\right) \neq$ $D_{K L}\left(t_{\text {max }}^{i+1} \| t_{\max }^{i}\right)$; therefore, it fulfills the assumption that the changes in SITS occur in chronological order.

\section{EXPERIMENTAL RESULTS AND DISCUSSION}

Throughout our experiments we address vegetation changes of three significant land-cover classes including two types of irrigated agriculture fields (Rice and Berries) and Marshlands. 


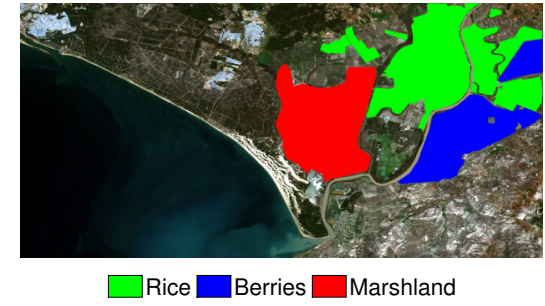

Fig. 1: Manual annotation of the target land-cover classes.

\begin{tabular}{|c|c|c|}
\hline Index & Landsat (2009/2010) & Sentinel-2 (2015/2016) \\
\hline \hline 1 & $23 \mathrm{Sept}-09 \mathrm{Oct}$ & $01 \mathrm{Dec}-12 \mathrm{Dec}$ \\
\hline 2 & $09 \mathrm{Oct}-02 \mathrm{Nov}$ & $12 \mathrm{Dec}-09 \mathrm{Mar}$ \\
\hline 3 & $02 \mathrm{Nov}-04 \mathrm{Dec}$ & $09 \mathrm{Mar}-08 \mathrm{Apr}$ \\
4 & $04 \mathrm{Dec}-29 \mathrm{Jan}$ & $08 \mathrm{Apr}-22 \mathrm{May}$ \\
\hline 5 & $29 \mathrm{Jan}-06 \mathrm{Feb}$ & $22 \mathrm{May}-07 \mathrm{Jun}$ \\
\hline 6 & $06 \mathrm{Feb}-03 \mathrm{Apr}$ & $07 \mathrm{Jun}-06 \mathrm{Jul}$ \\
7 & $03 \mathrm{Apr}-27 \mathrm{Apr}$ & $06 \mathrm{Jul}-20 \mathrm{Jul}$ \\
\hline 8 & $27 \mathrm{Apr}-05 \mathrm{May}$ & $20 \mathrm{Jul}-26 \mathrm{Jul}$ \\
\hline 9 & $05 \mathrm{May}-21 \mathrm{May}$ & $26 \mathrm{Jul}-08 \mathrm{Aug}$ \\
\hline 10 & $21 \mathrm{May}-06 \mathrm{Jun}$ & $08 \mathrm{Aug}-05 \mathrm{Sept}$ \\
\hline 11 & $06 \mathrm{Jun}-22 \mathrm{Jun}$ & $05 \mathrm{Sept}-04 \mathrm{Oct}$ \\
\hline 12 & $22 \mathrm{Jun}-30 \mathrm{Jun}$ & - \\
\hline 13 & 30 Jun $-16 \mathrm{Jul}$ & - \\
\hline 14 & $16 \mathrm{Jul}-01 \mathrm{Aug}$ & - \\
\hline 15 & $01 \mathrm{Aug}-25 \mathrm{Aug}$ & - \\
\hline
\end{tabular}

TABLE I: Time intervals corresponding to the indices of the plots in Fig. 2 and Fig. 3.

In order to analyze the change dynamics for these land-cover classes, a ground truth annotation was manually generated based on the information provided in [1], [8] and [10], which is shown in Fig. 1. The results were then validated based on the previous studies of land-cover changes in the Doñana National Park [1], [8], [10]-[13]. Moreover, the results were qualitatively evaluated by a group of experts, knowledgeable on the land-cover of the Doñana National Park.

Fig. 2 and 3 demonstrate the vegetation change dynamics in the three land-cover classes, where TABLE I depict the time intervals in which the changes were measured. Fig 2 (a) and (b) represent the computed change as $D_{K L}$ for the Landsat and Sentinel-2 datasets, respectively. In order to be comparable, the results were normalized by the time intervals. According to Fig. 2 (a), marshlands experience a significant change during winter (04 Dec - 06 Feb) which is due to flooding. This is consistent with the flooding cycle of the region reported in [11]. Comparing to the results obtained from the Landsat dataset, Sentinel-2 dataset does not show a considerable change in marshlands. According to [12], in 2015/2016 when the Sentinel-2 images were captured the area was fully covered by the flood water and the region experienced a dryer year as compared to 2009/2010 when the Landsat images were captured. Therefore, the range of the vegetation dynamics in $2009 / 2010$ was larger than that of in $2015 / 2016$.

Fig. 3 show the change dynamics for the target classes as the differences between NDVI values of consecutive images (blue curves) versus the change dynamics computed based on our approach (red curves). The first and second rows present the results based on the Landsat and Sentinel-2 datasets, respectively. The figure demonstrates that the change dynamics computed by our approach has similar patterns as the NDVI does in the most cases. Fig. 4 visualizes examples of the

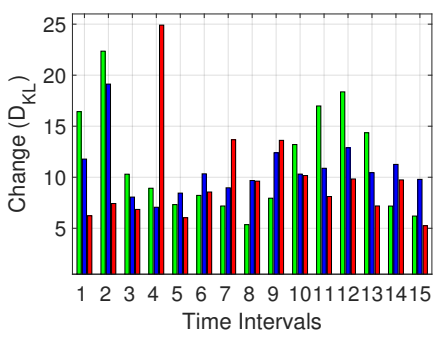

(a)

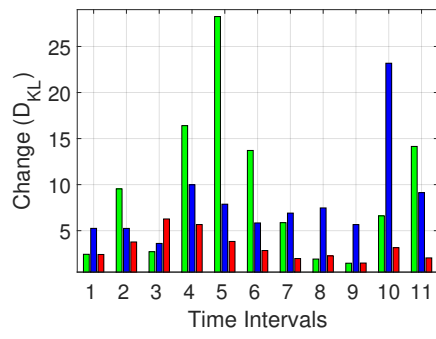

(b) $\square$ Rice $\square$ Berries $\square$ Marshland

Fig. 2: Changes computed based on the (a) Landsat and (b) Sentinel-2 datasets for the three target land-cover classes. The time intervals are depicted in TABLE I.

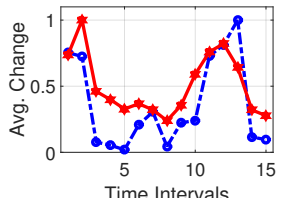

(a) Rice

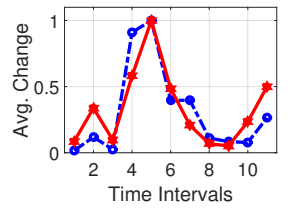

(d) Rice

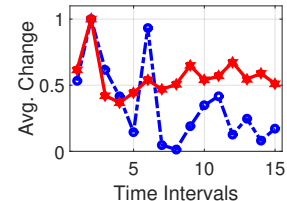

(b) Berries

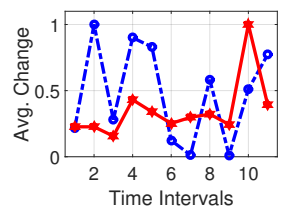

(e) Berries

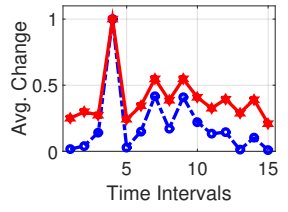

(c) Marshland

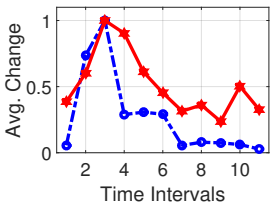

(f) Marshland
Fig. 3: Change dynamics as the differences between NDVI values (blue curves) versus the change dynamics based on our approach (red curves). (a-c) and (d-f) present results based on the Landsat and Sentinel-2 datasets, respectively. The time intervals are depicted in TABLE I.

vegetation changes computed by our approach. According to Fig. 3 (d), large changes in marshlands occurred between 04 Dec and 06 Feb which is also obvious in Fig. 4 (a). In February, the marshlands were covered by water and therefore, there was only slight change in the water borders according to Fig. 3 (d) and Fig. 4 (b). This means that the flood started draining and new vegetation was growing instead. In April and May, almost all the flooding water drained from the region and small plants were growing resulting a large degree of vegetation change in the marshlands, Fig. 3 (d) and Fig. 4 (c).

As the next example, we analyze the rice paddies' dynamics. According to Fig. 3 (a) and (d), from late May to early July there were significant changes in both datasets. This period is the beginning of the rice plantation seasons and therefore, according to [12], the vegetation mass increases steeply. However, after these periods, the change in the vegetation mass decreases exponentially until the rice grains become straw. As more grains turn yellow, the change exhibit a quadratic growth until the harvest time, from late September to late October. Fig. $4(\mathrm{~d}-\mathrm{f})$ and $(\mathrm{g}-\mathrm{i})$ visualize the vegetation changes in rice fields between May and August based on the Landsat and Sentinel-2 datasets, respectively. According to 


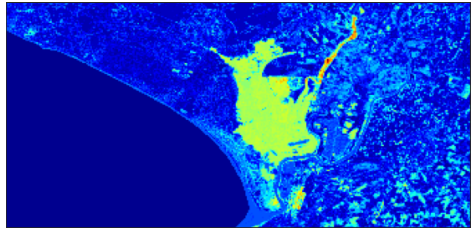

(a) 04 Dec - 06 Feb (Landsat)

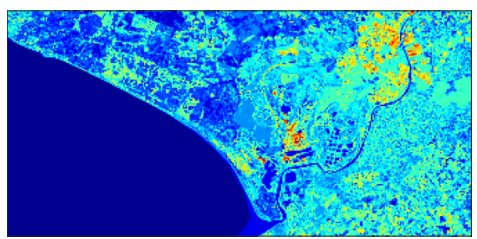

(d) 21 May - 06 Jun (Landsat)

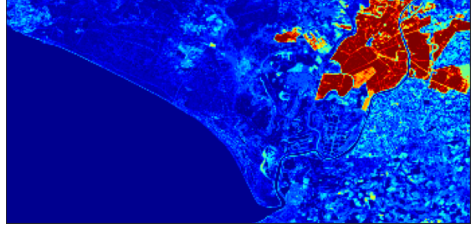

(g) 22 May - 07 Jun (Sentinel-2)

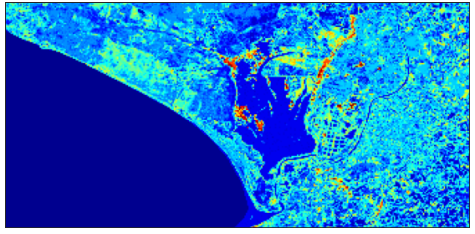

(b) 06 Feb - 03 Apr (Landsat)

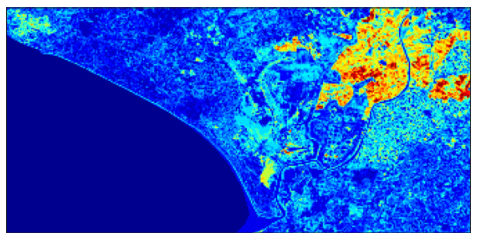

(e) 06 Jun - 30 Jun (Landsat)

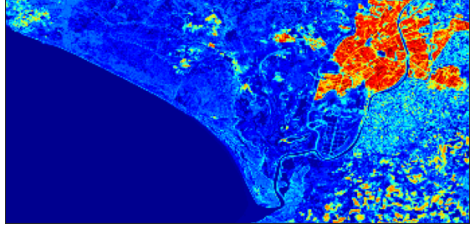

(h) 07 Jun - 06 Jul (Sentinel-2)

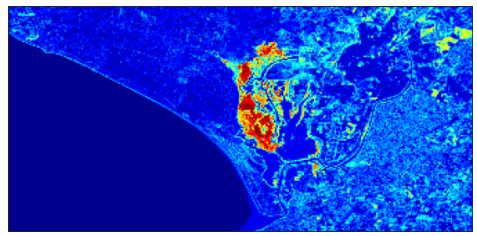

(c) 03 Apr - 05 May (Landsat)

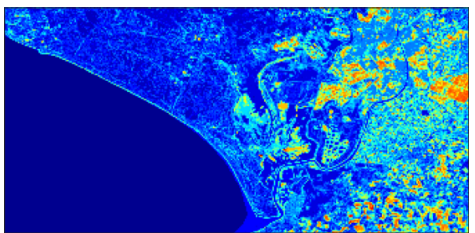

(f) 30 Jun - 01 Aug (Landsat)

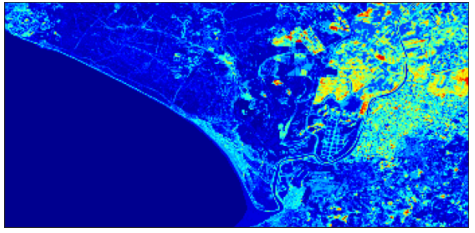

(i) 06 Jul - 08 Aug (Sentinel-2)

$\min$

Fig. 4: Demonstration of the vegetation changes computed by our proposed method. $(a-c)$ in marshlands between December and May based on Landsat, (d-f) in rice fields between May and August based on Landsat, and ( $\mathrm{g}-\mathrm{i}$ ) based on Sentinel-2.

Fig. 4 (d) and (g), some rice paddies experienced a considerable change in May 2010, whereas in May 2016 the whole rice plantation area largely changed. While in June 2010 the change increased in the rice paddies, in June 2015 the changes slightly decreased, as seen in Fig. 4 (e) and (h). In both years, the vegetation mass of the rice paddies experienced a gentle change in July, Fig. 4 (f) and (i). Additionally, comparing the computed changes in Fig. 4, the rice paddies were cultivated more homogeneously in 2015, as the whole area was changing similarly. According to Fig. 3 (b) and (d), the Landsat dataset shows a significant vegetation mass change in the berry fields in October 2009; however, according to the Sentinel-2 dataset, in 2016 the fields experienced this change in August.

\section{CONClusion}

In this paper, we proposed an approach for land-cover change analysis based on LDA. For our experiments we used a Landsat and a Sentinel-2 SITS dataset of the Doñana National Park. Results show that the changes measured by the proposed approach is able to represent the vegetation dynamics of the study area. Additionally, comparing the results obtained from the two datasets demonstrates that using highlevel information allows the proposed approach to measure the changes regardless of the sensor. This will help long-term landcover monitoring by combining various available data. For a future work, one can further analyze the changes measured by the topics based on other spectral indices.

\section{ACKNOWLEDGMENT}

This research was funded partially by the European Union's H2020 program under agreement No. 641762 to the ECOPO-
TENTIAL project (www.ecopotential-project.eu) and by the German Academic Exchange Service (DAAD).

\section{REFERENCES}

[1] Ricardo Díaz-Delgado, David Aragonés, Isabel Afán, and Javier Bustamante, "Long-term monitoring of the flooding regime and hydroperiod of doana marshes with landsat time series (1974-2014)," Remote Sens., vol. 8, no. 9, 2016.

[2] Y. Tan, B. Bai, and M. S. Mohammad, "Time series remote sensing based dynamic monitoring of land use and land cover change," in Proc. EORSA, July 2016, pp. 202-206.

[3] T. Costchioiu, R. Constantinescu, and M. Datcu, "Multitemporal satellite image time series analysis of urban development in Bucharest and Ilfov areas," in Proc. COMM, May 2014, pp. 1-4.

[4] ESA, "Esa declares end of mission for envisat," 2012.

[5] "Landsat science team: 2016 winter meeting summary," The Earth Observer, vol. 1, no. 1, pp. 19-23, May 2016.

[6] Y. Ye, C. He, and Z. Zhi, "Classification of time series of SAR images based on generative model," in Proc. IGARSS, July 2016, pp. 11621165.

[7] David M. Blei, Andrew Y. Ng, and Michael I. Jordan, "Latent dirichlet allocation," Journal of Machine Learning Research, vol. 3, pp. $993-$ 1022, 2003.

[8] I. Palomo, B. Martín-López, P. Zorrilla-Miras, D. García Del Amo, and C. Montes, "Deliberative mapping of ecosystem services within and around Doñana National Park (SW Spain) in relation to land use change," Reg. Environ. Chang., vol. 14, no. 1, pp. 237-251, 2014.

[9] S. Kullback and R. A. Leibler, "On information and sufficiency," The Annals of Mathematical Statistics, vol. 22, no. 1, pp. 79-86, Mar. 1951.

[10] Dalberg, "Saving Doñana from danger to prosperity," WWF Analysis, pp. 1-27, September 2016.

[11] F. Garcia-Novo and C. Marín Cabrera, Doñana: Water and Biosphere, Ministry of the Environment, Madrid, Spain, 2006.

[12] "Servidor de imágenes landsat y productos derivados de Donãna," http://venus.ebd.csic.es/imgs/, Accessed: 2017.

[13] D. Espinoza-Molina, R. Bahmanyar, R. Díaz-Delgado, J. Bustamante, and M. Datcu, "Land-cover change detection using local feature descriptors extracted from spectral indices," in Proc. IGARSS, July 2017. 